\title{
STATISTICAL COMPUTATIONS WITH ASTROGRID AND THE GRID
}

\author{
ROBERT NICHOL \\ Institute of Cosmology and Gravitation (ICG), Univ. of Portsmouth, Portsmouth, PO1 2EG, UK \\ GARRY SMITH \\ ICG Portsmouth 6 Institute of Astronomy, School of Physics, University of Edinburgh, UK. \\ CHRISTOPHER MILLER \\ Cerro-Tololo Inter-American Observatory, NOAO, Casilla 603, LaSerena, Chile \\ CHRIS GENOVESE, LARRY WASSERMAN \\ Dept. of Statistics, Carnegie Mellon University, Pittsburgh, PA-15213, USA \\ BRENT BRYAN, ALEXANDER GRAY, JEFF SCHNEIDER, ANDREW MOORE \\ School of Computer Science, Carnegie Mellon University, Pittsburgh, PA-15213, USA
}

\begin{abstract}
We outline our first steps towards marrying two new and emerging technologies; the Virtual Observatory (e.g, AstroGrid) and the computational grid. We discuss the construction of VOTechBroker, which is a modular software tool designed to abstract the tasks of submission and management of a large number of computational jobs to a distributed computer system. The broker will also interact with the AstroGrid workflow and MySpace environments. We present our planned usage of the VOTechBroker in computing a huge number of $\mathrm{n}$-point correlation functions from the SDSS, as well as fitting over a million CMBfast models to the WMAP data.
\end{abstract}

\section{Introduction}

Over a petabyte of raw astronomical data is expected to be collected in the next decade (see Szalay \& Gray 2001). This explosion of data also extends to the volume of parameters measured from these data including their errors, quality flags, weights and mask information. Furthermore, these massive datasets facilitate more complex analyses, e.g. nonparametric statistics, which are computationally intensive. A key question therefore is: Can existing statistical software scale-up to cope with such large datasets and massive calculations? We address this question here.

We focus here on two exciting new technologies, namely the Virtual Observatory (VO) and computational grids. However, we point the reader to Jim Linnemann paper in these proceedings for an excellent summary of existing statistical software packages in physics and astrophysics. We also direct the reader to the recent ADASS conference proceedings and the "Mining the Sky" proceedings (www.mpagarching.mpg.de/c̃osmo/).

\section{N-point Correlation Functions}

As a case study of the types of massive calculations planned for the next generation of astronomical surveys and analyses, we discuss here the galaxy n-point correlation functions. These have a long history in cosmology and are used to statistically quantify the degree of spatial clustering of a set of data points (e.g. galaxies). There are a hierarchy of correlation functions, starting with the 2-point correlation function, which measures the joint probability of a data pair, as a function of their separation $r$, compared to a Poisson distribution, i.e., $d P_{12}=N^{2} d V_{1} d V_{2}(1+\xi(r))$, where $d P_{12}$ is the joint probability of an object being located in both search volumes $d V_{1} \& d V_{2}$, and $N$ is the space density of objects. $\xi(r)$ is the 2-point correlation function and is zero for a Poisson distribution. If $\xi(r)$ is positive, then the objects are more clustered on scales of $r$ than expected, and vice versa for negative values.

The next in the series is the 3-point correlation function, which is defined as $d P_{123}=$ $N^{3} d V_{1} d V_{2} d V_{3}\left(1+\xi_{12}\left(r_{12}\right)+\xi_{23}\left(r_{23}\right)+\xi_{13}\left(r_{13}\right)+\right.$ $\left.\xi_{123}\left(r_{12}, r_{23}, r_{13}\right)\right)$, where $\xi_{12}, \xi_{12}, \xi_{12}$ are the 2-point functions for the three sides $\left(r_{12}, r_{23}, r_{13}\right)$ of the tri- 
angle and $\xi_{123}$ is the 3 -point function. Likewise, one can define a 4-point, 5-point etc., correlation function. The reader is referred to Peebles (1980) for a full discussion of these n-point correlation functions including their importance to cosmology (see also the recent lecture notes of Szapudi 2005). We also refer the reader to Landy \& Szalay (1993) and Szapudi \& Szalay (1998) for a discussion of the practical details of computing the $\mathrm{N}$-point functions.

Naively, the computation of the n-point correlation functions scale as $O\left(R^{n}\right)$, where $R$ is the number of data-points in the sample. As one can see, even with existing galaxy surveys from the Sloan Digital Sky Survey (SDSS), where $R \sim 10^{6}$ $10^{7}$, such correlation functions quickly become untractable to compute. In recent years, there has been a number of more efficient algorithms developed to beat this naive scaling. For example, the International Computational Astrostatistics (inCA; www.incagroup.org) group has developed a new algorithm based on the use of the multiresolutional KD-tree data structure (mrKDtrees). This software, known as npt, is publicly available (www.autonlab.org/autonweb/software/10378.html), and has been discussed previously in Gray et al. (2003), Nichol et al. (2001) and Moore et al. (2000). Briefly, mrKDtrees represent a condensed data structure in memory, which is used to efficiently answer as much of any data query as possible, i.e., pruning the tree in memory. The key advance of our $n p t$ algorithm is the use of " $\mathrm{n}$ " trees in memory together to compute an n-point function. See also Alex Gray's contribution in this volume.

\section{Computing Correlation Functions}

Even with an efficient algorithm, the computation of higher-order correlation functions is intensive. In detail, the $\mathrm{n}$-point correlation functions require a large number of sequential calls to the npt code. These include computing the cross-correlation between the real data (called $D$ ) and a random dataset (called $R$ ), which is used to mimic the edge effects in the real data. As outlined in Szapudi \& Szalay (1998), each estimation of a 3-point correlation functions, for a given bin of triangular shape (i.e., $r_{12} \pm \Delta_{r_{12}}, r_{23} \pm \Delta_{r_{23}}, r_{13} \pm \Delta_{r_{13}}$, requires seven separate source counts over the whole dataset, namely $D D D, D D R, D R R, R R R, D D, R R, D R$. Therefore,

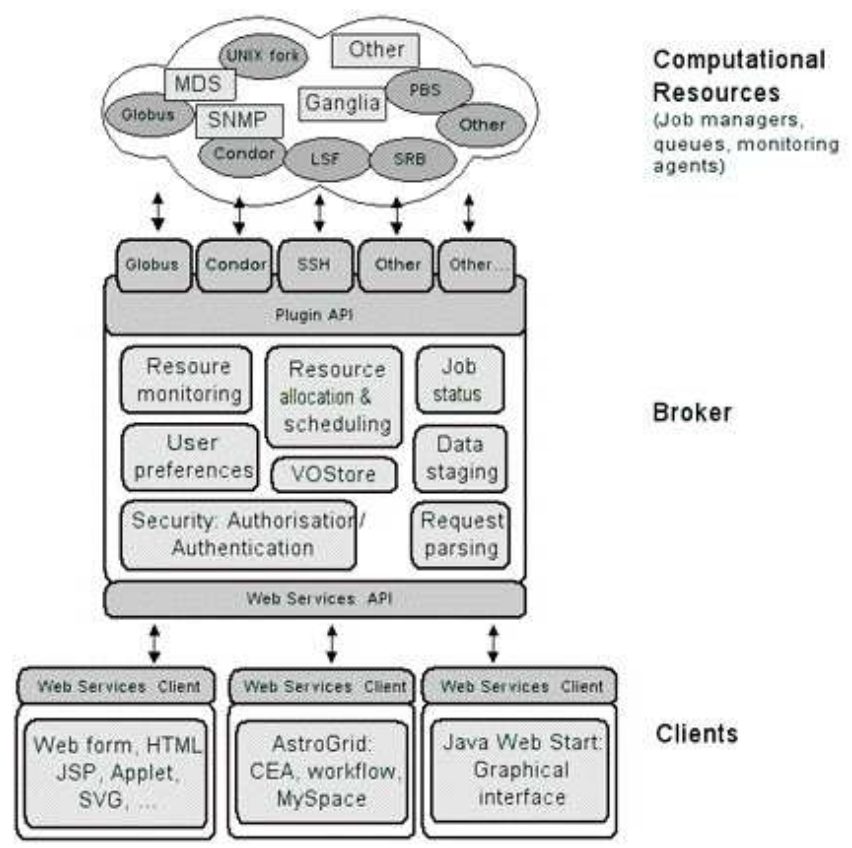

Fig. 1. The archtecture of the VOTechBroker and how it interacts with the Grid, VO and our statistical algorithms. The $n p t$ algorithm is a "Client" (at the bottom) and interacts with the "Broker" via a web-form (HTML) to define the basic parameters needed to run the algorithm and define the resources needed. Eventually we plan to interact with the "Broker" via the AstroGrid workflow environment, allowing the submission of jobs as well as the storage of the input data and results in MySpace. There can be multiple "Clients" to the "Broker".

if one wished to probe $\sim 10^{2}$ triangle configuration, then $\sim 10^{3}$ sequential $n p t$ jobs are required. This can rise rapidly if one wishes to estimate errors on the $\mathrm{n}$-point functions using either jack-knife resampling (i.e., removing subregions of the data and then recomputing the correlation functions), or a large ensemble of mock catalogs (derived from simulations). Such computations are well-suited to large clusters or grid of computers.

In recent years, we have used computational resources like TeraGrid (www.teragrid.org) and COSMOS (www.damtp.cam.ac.uk/cosmos/) to perform the computation of the $\mathrm{n}$-point correlation functions for the SDSS main galaxy sample and the SDSS LRG sample. Our experience shows that the management and scheduling of such a large number of jobs on these massive machines is laborious and tedious. To ease this problem, we are working on VOTechBro$k e r$, which is a tool that joins two new and emerging technologies; the VO and computational grids. 


\section{VOTechBroker}

AstroGrid (www.astrogrid.org) is a PPARC-funded project to create a working Virtual Observatory for UK and international astronomers. AstroGrid works closely with other VO initiatives around the world (via the International Virtual Observatory Alliance; IVOA) and is part of the Euro-VO initiative in Europe. In particular, the work outlined here has been performed as part of the EU-funded VOTech project, which aims to complete the technical preparation work for the construction of a European Virtual Observatory. Specifically, VOTech is undertaking R\&D into data-mining and visualization tools, which can be integrated into the emerging $\mathrm{VO}$ and computational grid infrastructure. Therefore, VOTech will build upon existing or emerging standards and infrastructure (e.g. IVOA standards and AstroGrid middleware), as well as looking at standards from W3C and GGF.

As part of the VOTech research, we are engaged in developing the VOTechBroker. The key design goals of the broker are to: i) Remove the execution and management of a large number of jobs (like $n p t)$ from the user in a transparent and reusable way; ii) Accommodate different grid infrastructures (e.g. condor, globus etc.); iii) Locate suitable resources on the grid and optimize the submission of jobs; iv) Monitor the status and success of jobs; v) Combine with AstroGrid MySpace and workflow environments to allow easy management of job submission and final results (as well as utilizing other algorithms within the VO). In Figure 1, we show the schematic design of the broker archtecture which illustrates the modular and "plug-in" design philosophy we have adopted. This is required as one of the key requirements of VOTechBroker is that it should be straightforward to add new algorithms, resources and middleware (e.g. a different job submission tool or protocol).

We have implemented the core functionality of VOTechBroker and are presently testing it by submitting $\sim 10^{4} n p t$ jobs on both the UK National Grid Servise (www.ngs.ac.uk), COSMOS supercomputer and a local condor pool of machines. The key ingredients of the present VOTechBroker include GridSAM (an open-source job submission and monitoring web servise from the London e-Science Centre), the UK e-Science X.509 certificates, MyProxy (a repository for X.509 Public Key Infrastructure security creden-

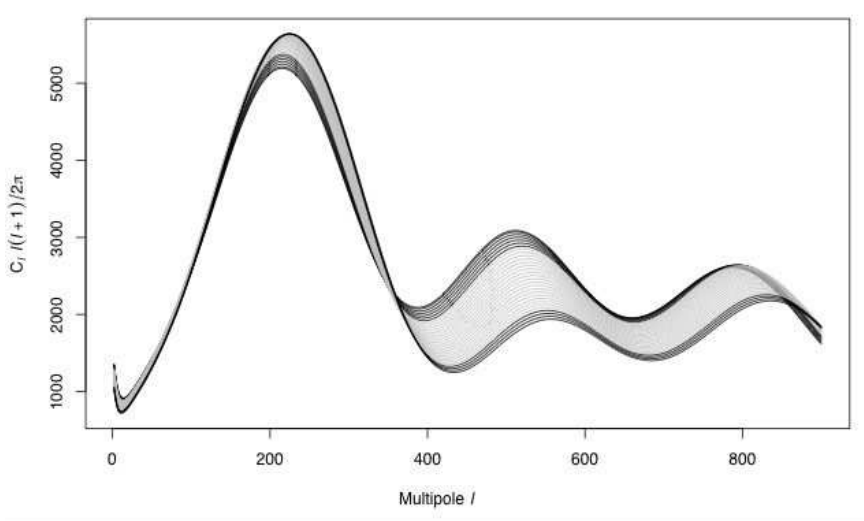

Fig. 2. Using CMBfast, we have varied $\Omega_{b}$ (baryon fraction) and determined which models lie within the $95 \%$ confidence ball around $f\left(X_{i}\right)$. For this illustration, we have kept all other parameters in these CMBfast models fixed at their fiducial values. The gray models are within the confidence ball, while the others are outside the ball indicating they are "bad fits" to the data (at the $95 \%$ confidence). We get an allowed range of $0.0169<\Omega_{b}<0.0287$.

tials), and the Job Submission Description Language (JSDL; a standard description of job execution requirements to a range of resource managers from the Global Grid Forum). At present, the VOTechBroker provides a web-form interface to just the npt algorithm discussed above but is modular in design so other algorithms can be easily added via other webforms. Results from the VOTechBroker will soon be placed in a user's AstroGrid MySpace. In the near future, we will interface the broker with other computational resources, e.g., TeraGrid (see below), and the AstroGrid workflow.

\section{Nonparametric Statistics}

In addition to the need for new statistical software that scales-up to petabyte datasets, we also require new algorithms and computational resources that exploit the emerging power of nonparametric statistics. As discussed in Wasserman et al. (2001), such nonparametric methods are statistical techniques that make as few assumptions as possible about the process that generated the data. Such methods are more flexible than more traditional parametric methods that impose rigid and often unrealistic assumptions. With large sample sizes, nonparametric methods make it possible to find subtle effects which might otherwise be obscured by the assumptions built into 
parametric methods.

In Genovese et al. (2004), we discuss the application of nonparametric techniques to the analysis of the power spectrum of anisotropies in the Cosmic Microwave Background (CMB). For example, one can ask the simple question: How many peaks are detected in the WMAP CMB power spectrum? This question is hard to answer using parametric models for the CMB (e.g. CMBfast models) as these models possess multiple peaks and troughs, which could potentially be fit to noise rather than real peaks in the data. To solve this, we have performed a nonparametric analysis of the WMAP power spectrum (Miller et al. 2003), which involves explaining the observed data $\left(Y_{i}\right)$ as $Y_{i}=f\left(X_{i}\right)+c_{i}$ where $f\left(X_{i}\right)$ is a orthogonal function (expanded as a cosine basis $\left.\beta_{i} \cos \left(i \pi X_{i}\right)\right)$ and $c_{i}$ is the covariance matrix. The challenge is to "shrink" $f\left(X_{i}\right)$ to keep the number of coefficients $\left(\beta_{i}\right)$ to a minimum. We achieve this using the method of Beran (2000), where the number of coefficients kept is equal to the number of data points. This is optimal for all smooth functions and provides valid confidence intervals. We also use monotonic shrinkage of $\beta_{i}$, specifically the nested subset selection (NSS). The main advantage of this methodology is that it provides a "confidence ball" (in N dimensions) around $f\left(X_{i}\right)$, allowing non-parametric interferences like: Is the second peak in the WMAP power spectrum detected? In addition, we can test parametric models against the "confidence ball" thus quickly assessing the validity of such models in $\mathrm{N}$ dimensions. This is illustrated in Figure 2.

\section{Massive Model Testing}

We are embarked on a major effort to jointly search the 7-dimensional cosmological parameter-space of $\Omega_{m}, \Omega_{D E}, \Omega_{b}, \tau$, neutrino fraction, spectral index and $\mathrm{H}_{0}$ using parametric models created by CMBfast and thus determine which of these models fit within the confidence ball around our $f\left(X_{i}\right)$ at the $95 \%$ confidence limit. Traditionally, this is done by marginalising over the other parameters to gain confidence intervals on each parameter separately. This is a problem in high-dimensions where the likelihood function can be degenerate, ill-defined and under-identified. Unfortunately, the nonparametric approach is computational intense as millions of models need to searched, each of which takes $\simeq 3$ minute to run.
To mitgate this problem, we have developed an intelligent method for searching for the surface of the confidence ball in high-dimensions based on Kriging. Briefly, kriging is a method of interpolation which predicts unknown values from data observed at known locations (also known as Gaussian process regression, which is a form of Bayesian inference in Statistics). There are many different metrics for evaluating the kriging success; we use here the "Straddle" method which picks new test points based both on the overall distance from previous searched points, as well as being predicted to be close to the boundary of the confidence ball. We have also developed a heuristic algorithm for searching for "missed peaks" in the likelihood space by searching models along the path joining previously detected peaks. We find no "missed peaks", which illustrates our kriging algorithm is effective in finding the surface of the confidence ball in this high dimensional space.

We have distributed the CMBfast model computations over a local condor pool of computers. In Figure 3, we show preliminary results from this high-dimension search for the surface of the confidence ball and present joint $2 \mathrm{D}$ confidence limits on pairs of the aforementioned cosmological parameters. These calculations represent 6.8 years of CPU time to calculate over one million CMBfast models. In the near future, we will move this analysis to TeraGrid, using VOTechBroker, and plan 10 million models to fully map the surface of the confidence ball. We will also make available a Java-based web servise for accessing these models, and the WMAP confidence ball, thus allowing other users to rapidly combine their data with our WMAP constraints e.g., doing a joint constraint from LSS and CMB data. We are also working on possible convergence tests, and visualization tools within VOTech, to access this highdimensional data.

\section{Summary}

The two examples given here - massive model testing of the WMAP data using nonparametric statistics and higher-order correlation functions of SDSS galaxies - represent a growing trend in astrophysics and cosmology for massive statistical computations. Our plan is to develop the VOTechBroker to provide a power framework within which such massive astronomical analyse can be performed. As discussed, 

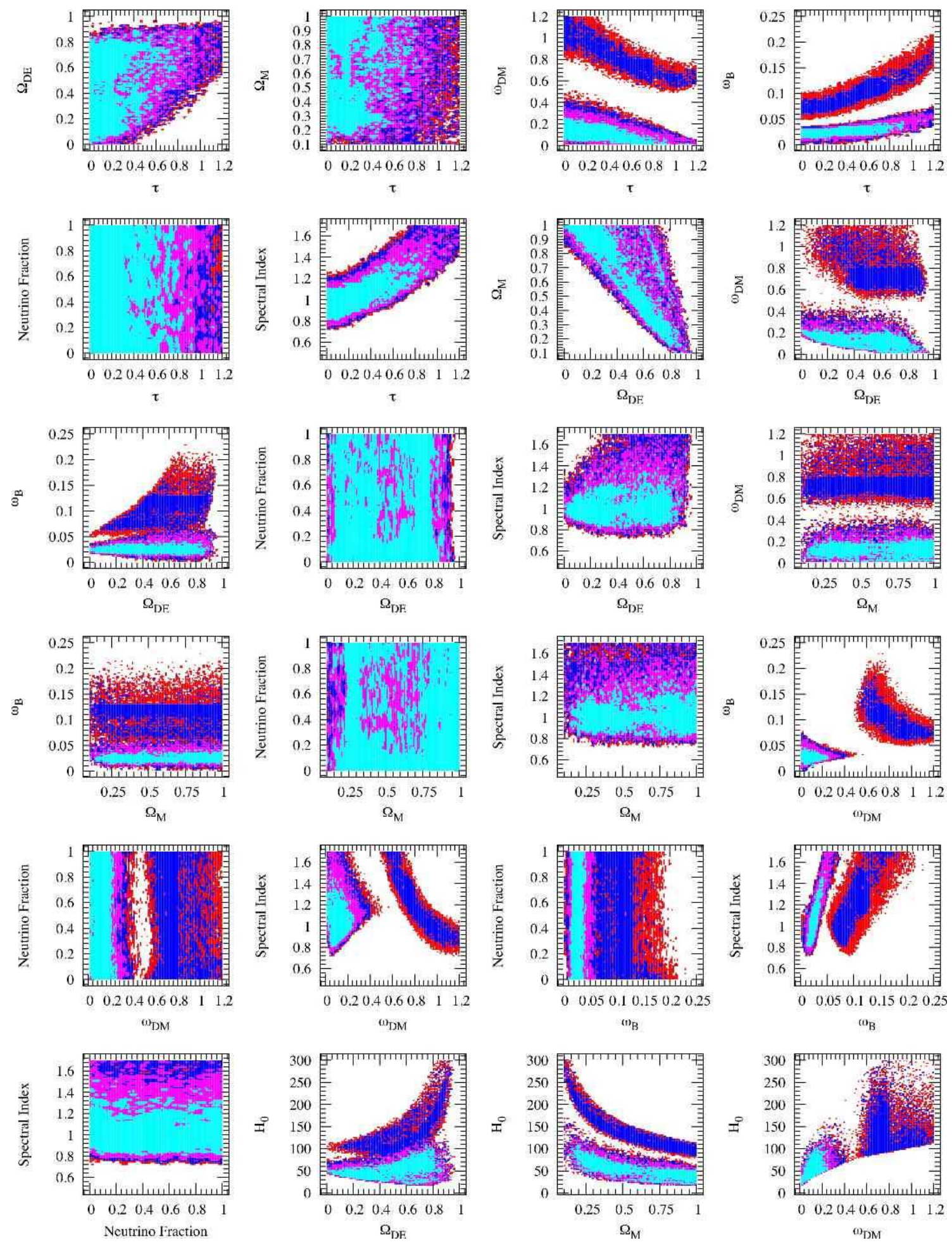

Fig. 3. The results of our 7-dimensional parameter search using 1.2 million models from CMBfast. The light blue (or lightest shading for greyscales) color are models excluded at the $34 \%$ level. The purple (or mid-grade shading) are models excluded by the $68 \%$ confidence ball and the red is the $95 \%$ confidence ball 
the complexities of job submission and management on computational grids, as well as being a modular "plug-in" design so other algorithms and software can be easily added. Finally, we plan to integrate VOTechBroker into the AstroGrid workflow and MySpace environments, so it becomes a natural repository for a host of advanced statistical algorithms than scale-up in preparation for petabytescale datasets and analyses.

\section{Acknowledgments}

We thank all my collaborators and colleagues in inCA, VOTech, AstroGrid, SDSS and VO projects. The work presented here was partly funded by NSF ITR Grant 0121671 and through the EU VOTech and Marie Curie programs. RCN thanks the organisers of the Phystat2005 meeting for their invitation. GS thanks the VOTech and University of Edinburgh for his funding (see eurovotech.org for details).

\section{References}

1. Beran, R. J. Amer. Stat. Assoc. 95, 155 (2000).

2. Genovese, C., et al., Statistical Science, (2004), astro-ph/0410140

3. Gray, A., et al. Conference Proceeding for ADASS XIII, (2003), astro-ph/0401121

4. Landy, S. D. \& Szalay, A., Astrophysical Journal 412, 64 (1993).

5. Miller, C. J., et al. Astrophysical Journal 565, 67 (2002).

6. Moore, A. W., et al. Conference Proceeding for "Mining the Sky", (2000), astro-ph/0012333

7. Nichol, R. C., et al. Conference Proceedings for "Statistical Challenges in Modern Astronomy III",(2001), astro-ph/0110230

8. Peebles, P. J. E., Large-scale structure in the Universe, Princeton University Press, (1980)

9. Szalay, A. \& Gray J., Science 293,, 2037, (2001).

10. Szapudi, I. (2005), astro-ph/0505391

11. Szapudi, I. \& Szalay, A., Astrophysical Journal 494, 41 (1998).

12. Wasserman, L., et al. Conference Proceedings for "Statistical Challenges in Modern Astronomy III",(2001), astro-ph/0112050 